\title{
The Role of Modified Radical Hysterectomy in Endometrial Carcinoma
}

\author{
Masamichi Hiura and Takayoshi Nogawa \\ Shikoku Cancer Center, \\ Japan
}

\section{Introduction}

Improvement of the treatment results in patients with endometrial carcinoma has been achieved by a multidisciplinary approach including surgery, chemotherapy and radiotherapy, similar to the case for many other carcinomas. Although total hysterectomy/bilateral salpingo-oophorectomy (TH/BSO), pelvic and para-aortic lymphadenectomy and peritoneal cytology are often required, cases with early carcinomas predominate among cases of endometrial carcinoma, and TH/BSO alone is sufficient to achieve a favorable prognosis in such cases. The recommended surgical procedure for the staging of a patient with endometrial carcinoma clinically confined to the fundal portion of the uterus includes peritoneal cytology and TH/BSO with pelvic and para-aortic lymphadenectomy (National Comprehensive Cancer Network, 2011). For operable patients with cervical involvement, peritoneal cytology and radical hysterectomy/bilateral salpingooophorectomy with pelvic and para-aortic lymphadenectomy should be considered. However, radical hysterectomy always has a major adverse effect with dysuria. Improvement of the treatment results and quality of life (QOL) can also be expected from modified radical hysterectomy performed in appropriately selected patients. One of the advantages of modified radical hysterectomy is that it can be switched from radical hysterectomy in high-risk patients in terms of the age, obesity and presence of medical complications. For operable patients with intra-abdominal disease, surgical procedure includes peritoneal cytology, TH/BSO with pelvic and para-aortic lymphadenectomy, and maximal debulking. The pathologic information obtained also provides an optimal basis for the selection of adjuvant therapy. Therefore, complete surgical staging including pathologic and prognostic data on which to base decisions regarding adjuvant therapy should be required for all patients who do not have medical or technical contraindication to lymphadenectomy.

There is no definitive data regarding the effectiveness of adjuvant chemotherapy in patients with uterine confined or intra-abdominal disease. The role of adjuvant chemotherapy in invasive high-grade tumor confined to the uterine body is the subject of current studies. Postoperative radiotherapy for patients with intermediate risk or high risk early stage endometrial carcinoma has been performed to prevent locoregional recurrence, however it did not increase overall survival (Creutzberg et al., 2000; Keys et al., 2004; Blake et al., 2008; Nout et al., 2010). Despite a traditional use of adjuvant radiotherapy, stage I/II patients with 
high risk factors may have a compromised survival due to extrapelvic metastatic disease, suggesting the need for effective systemic adjuvant therapy. Adjuvant chemotherapy may reduce the rate of distant recurrence in endometrial carcinoma. Therefore, it is reasonable to consider adjuvant chemotherapy for high risk endometrial carcinoma. Abdominal total hysterectomy is a basic gynecological surgical technique that beginners should try and master, and it is generally an extrafascial procedure. After obtaining full mastery over total hysterectomy, gynecologists in training should proceed to modified radical type II hysterectomy (Bidus \& Elkas, 2007; Jones. 2008; Randall et al., 2009), a procedure positioned in between total hysterectomy and radical hysterectomy, with or without lymphadenectomy This form of surgery is indicated for stage Ia1-Ia2 uterine cervical carcinoma and stage Ib-Ic, $\mathrm{IIa}$, and IIb (with slight infiltration of the cervical stroma) endometrial carcinoma. Complications of this procedure include bleeding and damage to the intestines, ureter and bladder. Due caution is necessary to avoid these complications or organ injuries, and it is important to extirpate the uterus together with the cardinal ligaments and to remove the vaginal wall with an extra $1.5-2.0 \mathrm{~cm}$ margin. There is uncertainty as to whether modified radical hysterectomy in high-risk endometrial carcinoma reduces locoregional recurrence. So, we performed modified radical type II hysterectomy including systematic pelvic and para-aortic lymphadenectomy in 284 stage I-IV endometrial carcinomas to prevent locoregional recurrence, patients with high risk factors (stages IC/II/III/IV) were treated by adjuvant chemotherapy.

The purpose of this study is to assess the role of modified radical hysterectomy in endometrial carcinoma, and identify the multivariate independent recurrence risk factors during past 10 years.

\section{Modified radical hysterectomy (Hiura \& Nogawa, 2011)}

In our method of modified radical type II hysterectomy, the anterior procedure following development of the paravesical and pararectal spaces involves retraction of the ureter in a lateral direction after dissecting the anterior layer of the vesicouterine ligament. However, the posterior layer of the vesicouterine ligament is not seprated. As the posterior procedure, the cardinal ligament is clamped en bloc with the posterior layer of the vesicouterine ligament and the deep layer of the sacrouterine ligament after dissecting the sacrouterine ligament, and the vaginal wall is resected with an extra $1.5-2.0 \mathrm{~cm}$ margin. The uterus is resected by dividing as much as possible the anterior uterine support and vaginal wall from the cervix. Another characteristic of this technique is that more of the cardinal ligament is resected compared with that in a total hysterectomy.

\subsection{Preoperative tests}

1. Hemoglobin, blood type, irregular antibody screening, serum chemistry, tests for infections, blood coagulation profile, blood glucose, and urinalysis.

2. Electrocardiography, lung function testing, and plain chest radiography.

3. Cervical cytology and endometrial cytology.

4. Magnetic resonance imaging (MRI), Computed tomography (CT), and Positron emission tomography-computed tomography (PET-CT): Patients with endometrial carcinoma should be examined for muscular infiltration by contrast-enhanced MRI. 
Contrast-enhanced CT is useful for evaluation of lymph node metastasis. PET-CT is also used for whole-body scanning when distant metastasis is suspected.

\subsection{Informed consent}

Informed consent is an extremely important element in establishing a trust relationship with the patient and her family. At our outpatient clinic, patients are provided with a full explanation at the time of their initial visits to the hospital, at the end of the examinations, and at the time of admission. Definitive written consent carrying the signature of the patient is obtained at the time of admission of the patient after following our institutional procedures in respect of giving information about the surgery and obtaining consent in the presence of the patient's family and a nurse. This is that part of a research protocol.

\subsection{Practical features of modified radical hysterectomy}

The operator basically stands on the left side, and the assistant on the right side of the patient. Intrapelvic procedures are easier from the left side for a right-handed operator. At laparotomy, the operator should wear a cotton glove on the left hand to avoid slippage when gently displacing and pulling the intestinal tract or the peritoneum. It is important for successful peritoneal procedures to obtain an adequate operative field, and incision of the abdominal wall allowing a 1-2 $\mathrm{cm}$ margin is an important point to this end. At our institution, in order to minimize postoperative pain, this surgery is performed under general anesthesia combined with epidural anesthesia, and an electric knife is used for every cutting procedure except for the hypogastric median skin incision. After laparotomy, the presence/absence of intraperitoneal lesions is examined by palpation and visual inspection, and the intraperitoneal site subject to surgical manipulation is observed carefully with the use of a lateral blades laparotomy retractor. With a full understanding of the positional relationships among the uterus, adnexa, intestinal tract and bladder, the operator elevates the small intestine from the minor pelvic cavity to the epigastric region, and compresses the left, right and central parts with gauze towels to prevent slippage of the small intestine. Then, a upper blade is set at the center to allow a sufficient operative field, and the surgical procedures are begun on the right side, proceeding thereafter to the left side. A flow-chart is given to illustrate the patient selection of modified radical hysterectomy for endometrial carcinoma (Fig. 1).

\subsubsection{Holding the uterus}

The uterus, adnexa and adjacent organs are palpated to confirm their positional relationships, and the degree of fixation and mobility. The round ligaments of the uterus, oviducts and ligaments of the ovary are clamped bilaterally with two long and straight Kocher forceps. The clamps are fixed firmly with gauze, and pulled to allow initiation of the manipulations from the right side.

\subsubsection{Clamping, cutting and ligation of the infundibulopelvic ligament}

To prevent ureteral injury, the infundibulopelvic ligament is clamped with two long and straight Kocher forceps after confirming the course of the ureter, while a short Kocher 
Preoperative tests

(1) Hemoglobin, blood type, irregular antibody screening, serum chemistry, tests for infections, blood coagulation profile, blood glucose, and urinalysis.

(2) Electrocardiography, lung function testing, and plain chest radiography.

(3) Cervical cytology and endometrial cytology.

(4) Magnetic resonance imaging(MRI), Computed tomography(CT), and Positron emission tomographycomputed tomography (PET-CT).

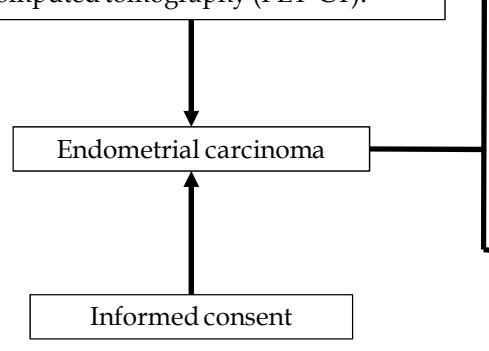

Indication of operation

Extrafascial procedure Endometrial carcinoma Stage Ib-Ic, IIa-IIb

Avoidance of complication (Bleeding, Damage to the intestines, ureter and bladder.)

- Disease confined to the uterine body

- Slight infiltration of the

cervical stroma

- Age

- Obesity

- Medical complication

Fig. 1. Flow-chart of the patient selection for modified radical hysterectomy in endometrial carcinoma.

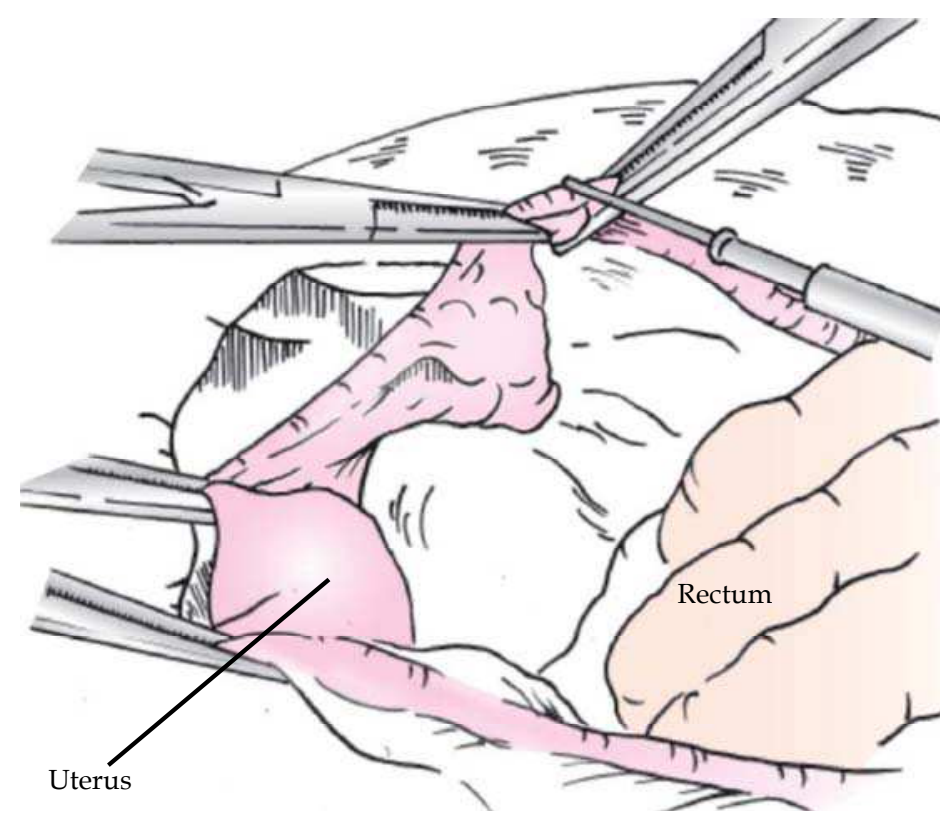

Fig. 2. Clamping, cutting and ligation of the infundibulopelvic ligament. 
forceps is set for the infundibulopelvic ligament to be preserved in order to prevent slippage. The infundibulopelvic ligament is then cut and ligated with 1-0 silk suture to be held, and pulled (Fig. 2). At this point, ureteral injury can be avoided if the infundibulopelvic ligament is clamped with a long and straight Kocher forceps while elevating the infundibulopelvic ligament with forceps with some distance maintained from the ureter. Opening the broad ligament close to the round ligament of the uterus makes the subsequent processing of the round ligament easier. Because there is the possibility of hydrosalpinx and fallopian tube cancer, the mesosalpinx on the fallopian tube side should be coagulated, incised and resected with an electric knife.

\subsubsection{Clamping, cutting and ligation of the round ligament of the uterus}

The round ligament of the uterus is clamped, cut, ligated with 1-0 silk suture, and pulled, while setting two long and straight Kocher forceps at a position 2/3 lateral to the uterus (Fig. 3). The uterus is pulled upward, detached and incised with an electric knife from the anterior lobe of the broad ligament toward the peritoneum of the vesicouterine pouch. Because webby thin and sparse connective tissue appears on the inner side of the peritoneum, manipulation of the electric knife as though using Cooper scissors is necessary for the detachment procedure. When incising the bladder peritoneum, approximating too close to the bladder would cause bleeding, therefore, caution is required.

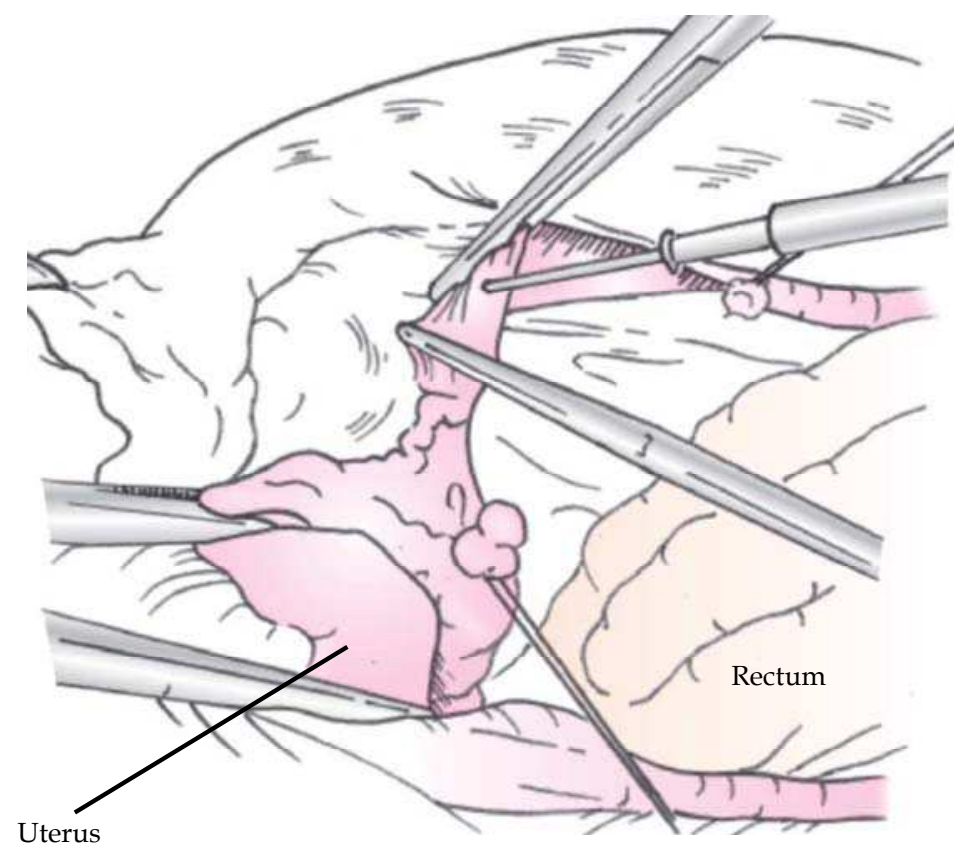

Fig. 3. Clamping, cutting and ligation of the round ligament of the uterus. 


\subsubsection{Development of the pararectal and paravesical spaces (in cases of pelvic lymphadenectomy)}

While the uterus is pulled left anteriorly, the assistant clamps and pulls the posterior lobe of the broad ligament with the straight Pean forceps to confirm the course of the ureter. The pararectal space in the pelvic floor is displaced in a left inguinal direction with a side plate under the course of the ureter attached to the posterior lobe of the broad ligament, and simultaneously, the non-resistive sparse connective tissue is displaced in 1-2 installments with Cooper scissors toward 180-degree opposite side. By this procedure, the pararectal space can be developed easily (Fig. 4). If there is resistance, the internal iliac artery may be compressed, and forced development may cause bleeding. Beginners should be particularly cautious about this point. Next, when the curved Pean forceps set for the bladder peritoneum are pulled, a funicular structure can be found in the connective tissue running toward the paravesical space. This structure is the paraumbilical ligament. When nonresistive sparse connective tissue is expanded with a side plate and Cooper scissors on the right and left side of the paraumbilical ligament, the paravesical space appears (Fig. 5). If a vascular tape is set for the paraumbilical ligament and fixed with a short Kocher forceps, subsequent manipulations become easier. Development of the pararectal and paravesical spaces should be done by a side plate and Cooper scissors. Displacing non-resistive sparse connective tissue in 1-2 installments would allow easy development. If there is resistance, the direction may be wrong, and should therefore be rechecked. The procedural steps for pelvic lymphadenectomy are skipped in this paper.

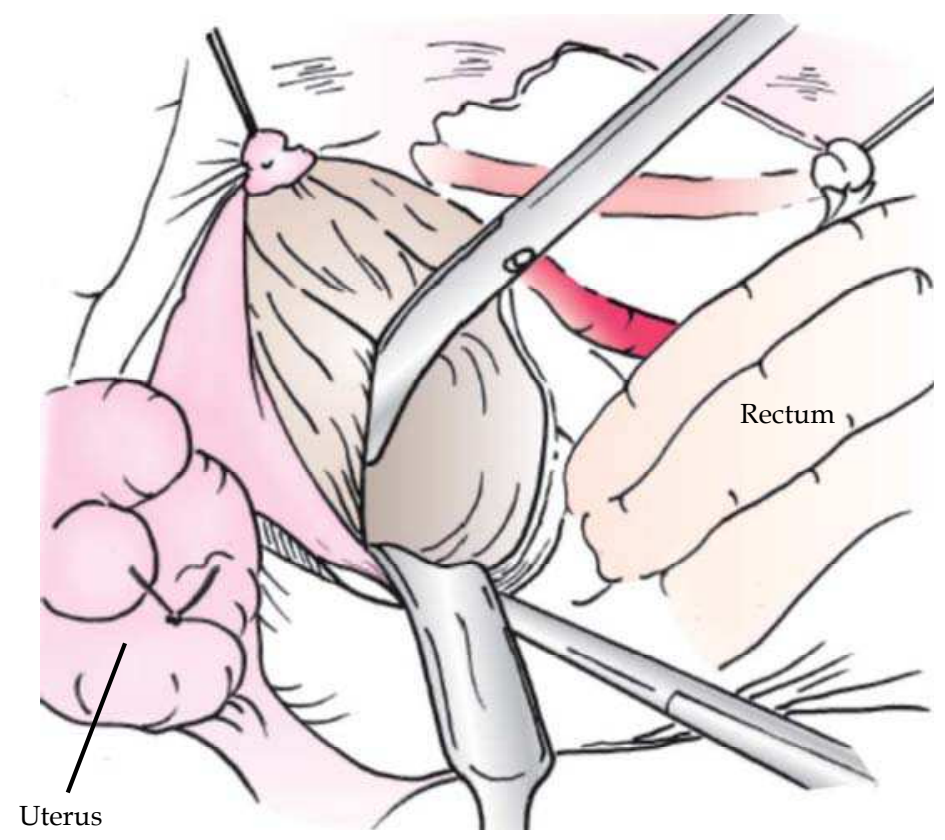

Fig. 4. Development of the pararectal space. 


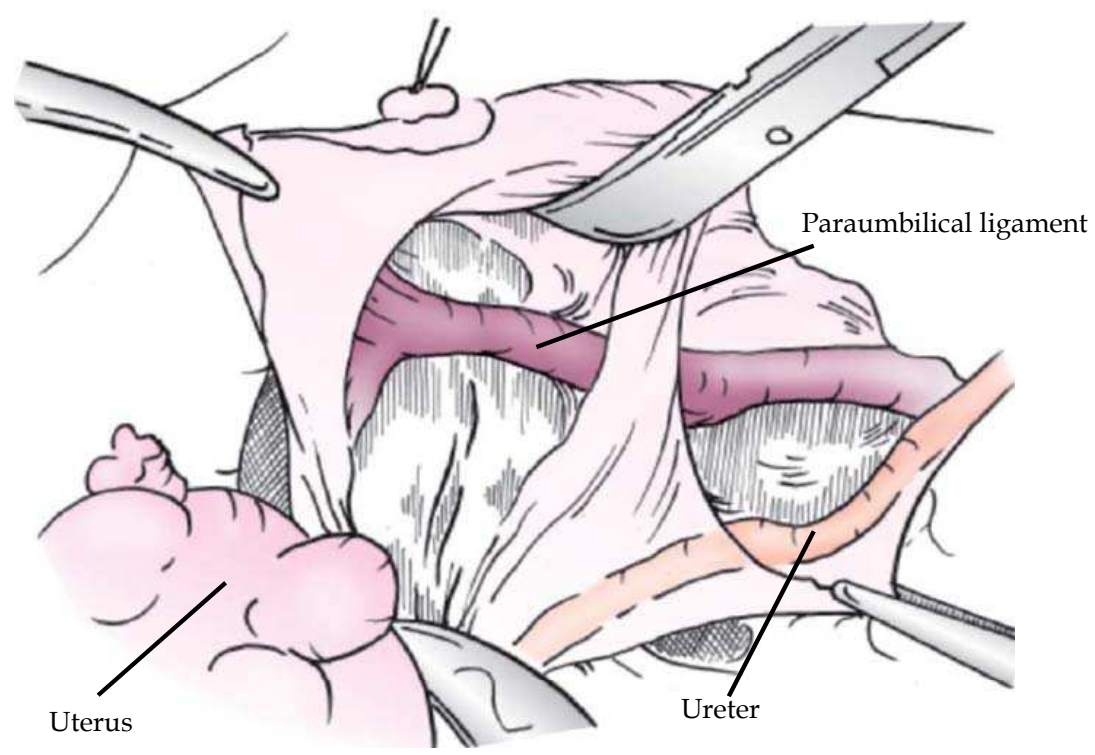

Fig. 5. Development of the paravesical space.

\subsubsection{Detachment of the ureter and cutting of the posterior lobe of the broad ligament}

After confirming the course of the ureter while pulling the posterior lobe of the broad ligament clamped with the straight Pean forceps, the ureter is isolated from the uterus by detaching it with Cooper scissors up to the vicinity of the right uterine artery. The knack for easier isolation of the ureter from the posterior lobe of the broad ligament involves detachment of the ureter with Cooper scissors first at an angle perpendicular to the course of the ureter and then along the course of the ureter (Fig. 6). Then, to separate the ureter from the uterus, the ureter is displaced laterally with a side plate, and the posterior lobe of the broad ligament is detached with an electric knife toward the vicinity of the sacrouterine ligament and the superficial layer, and then cut and developed. We commonly use a side plate because this surgical instrument is very useful for expansion of the operative field if the direction is properly determined on a side plate.

\subsubsection{Cutting of the sacrouterine ligament and opening of the Douglas' pouch}

While the assistant is pulling the uterus to make a 90-degree angle to the right sacrouterine ligament, the rectum and right pelvic peritoneum are tensed with the left hand, and the superficial layer of the right sacrouterine ligament is cut with an electric knife without ligation (Fig. 7).Then, while the assistant is pulling the uterus, the rectum is tensed with the left hand, and the Douglas' pouch is incised. After incising the thin funicular sparse 


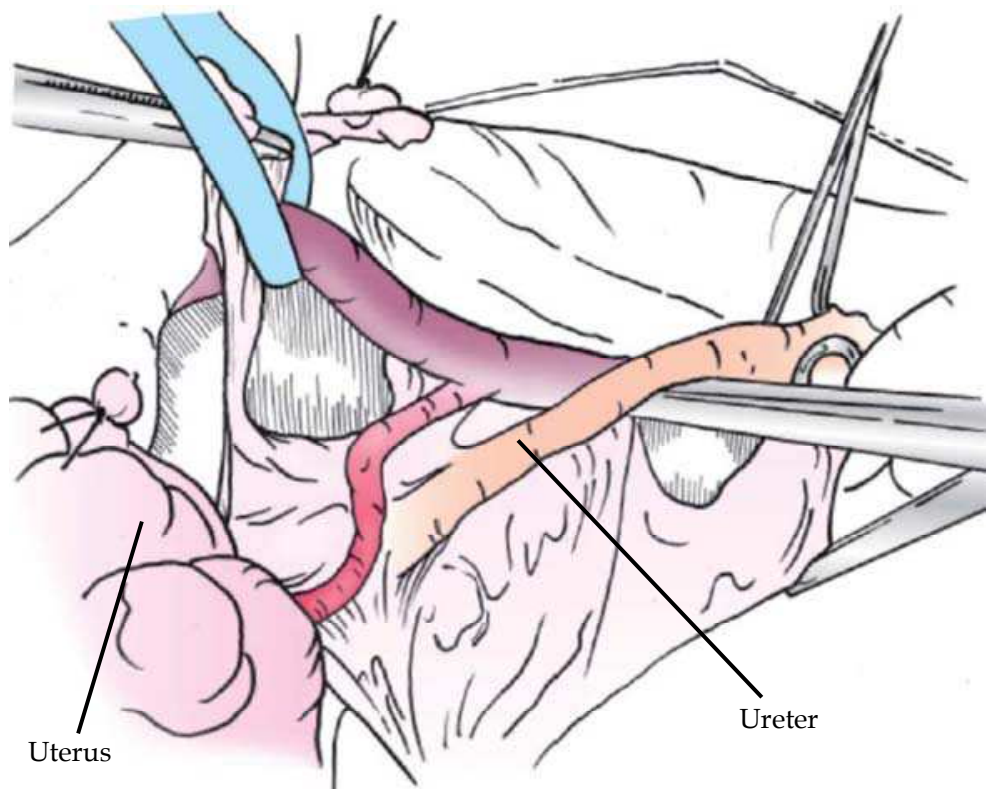

Fig. 6. Detachment of the ureter.

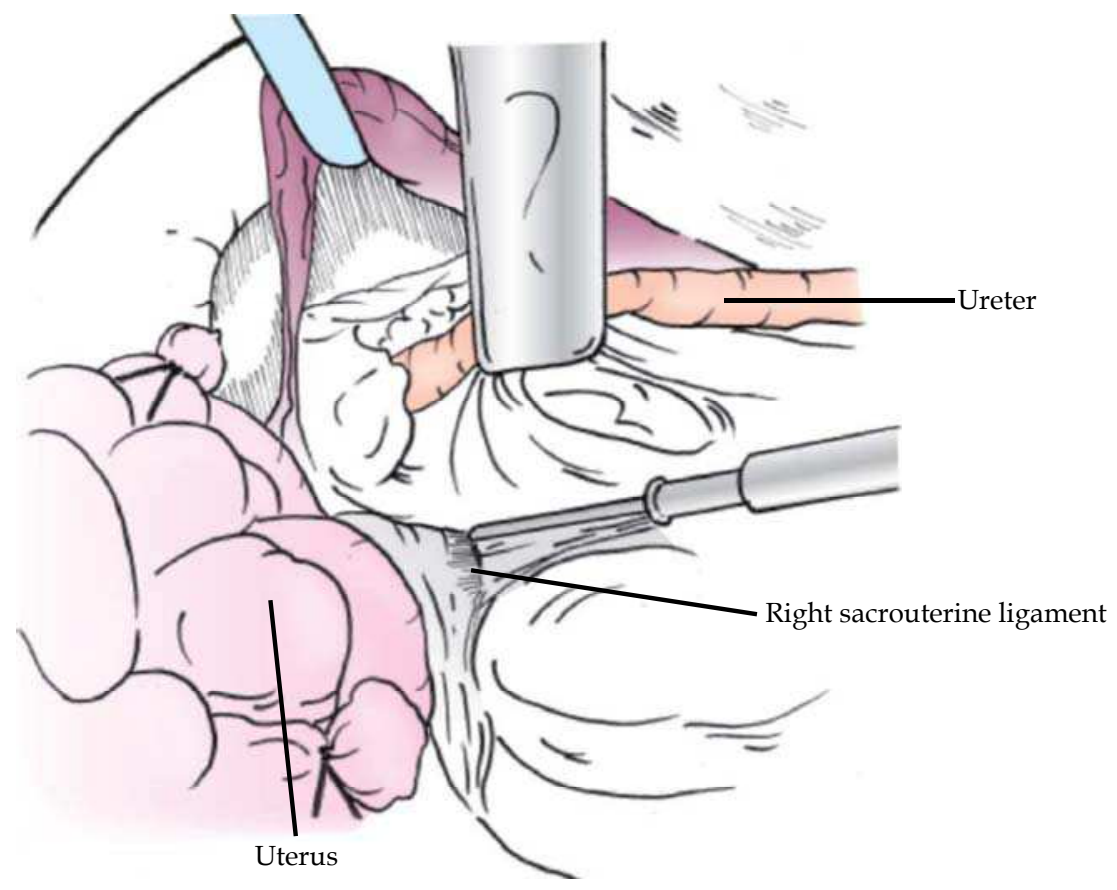

Fig. 7. Cutting of the sacrouterine ligament. 
connective tissue with an electric knife, the pouch opens spontaneously (Fig. 8). While avoiding injury to the hypogastric nerve, the deep layer of the sacrouterine ligament is detached and cut. When gauze is placed between the rectum and the vaginal wall and displaced downward with the fingers, the rectum can be detached easily from the vaginal wall. At this time, for a better result, the superficial layer and the posterior layer of the thin and funicular sacrouterine ligament, firmly tensed with the hand wearing a cotton glove to avoid slippage of the rectum and right pelvic peritoneum, should be incised little by little.

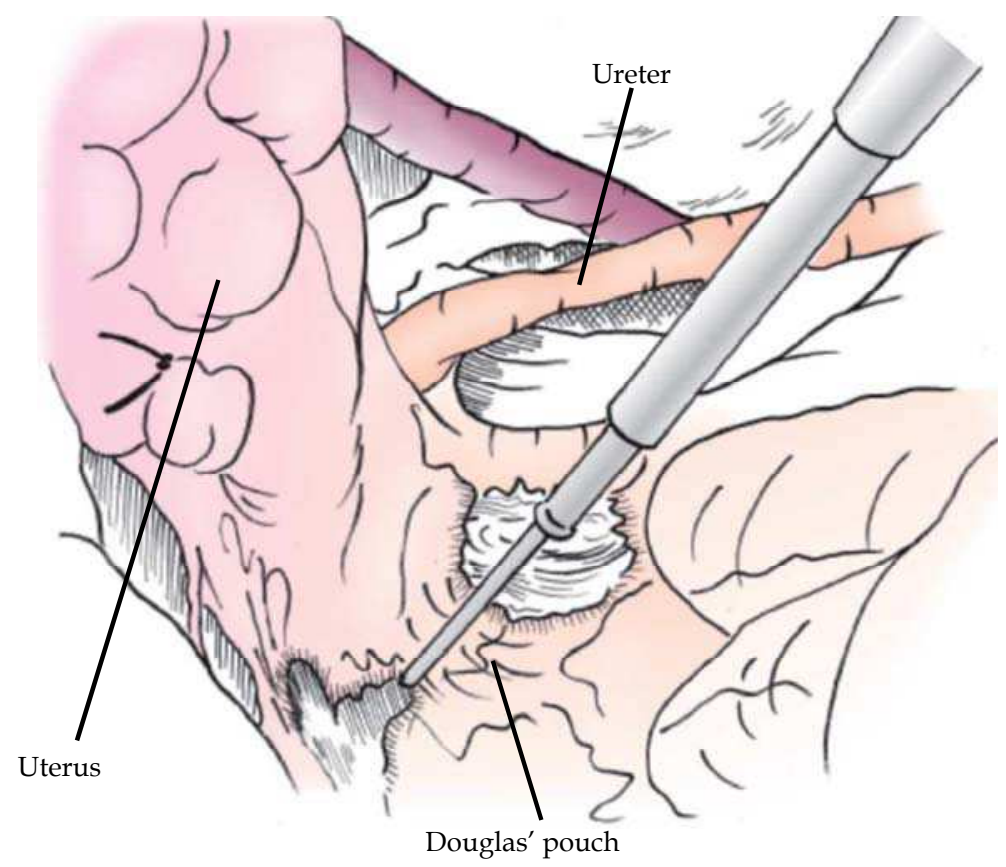

Fig. 8. Opening of the Douglas' pouch.

\subsubsection{Detachment of the bladder}

After similar manipulations on the left side, the operation proceeds to the process of detachment of the bladder. The uterus is elevated upward, and the positional relationship between the uterine cervix and the bladder is confirmed by palpation. Then, a small incision is made in the tensed vesicouterine pouch with an electric knife while the bladder peritoneum is elevated with the long and straight Pean forceps. This procedure causes the appearance of the bladder wall, the lustrous muscular layer of the uterine cervix, and the webby sparse connective tissue (Fig. 9).Gauze is placed in these structures, and the bladder is displaced and detached sufficiently with the gauze while manipulating it as though rolling it over downward with a side plate and Cooper scissors (Fig. 10). The bladder is detached and isolated at the center, and thereafter the sparse connective tissues on the left and right sides are detached with the Cooper scissors while pushing laterally. This procedure allows easy detachment of the bladder with scarcely any bleeding. If the sparse connective tissue on the lateral side of the uterus is sufficiently cut with an electric knife up 


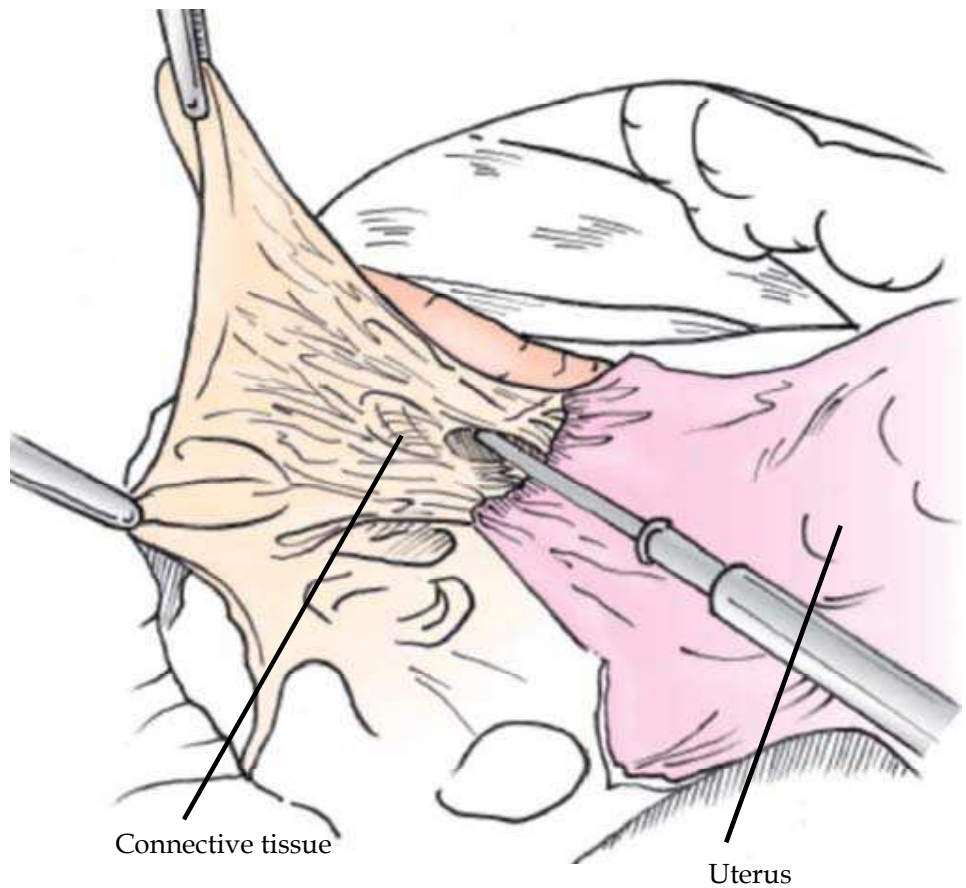

Fig. 9. Detachment of the urinary bladder (1)

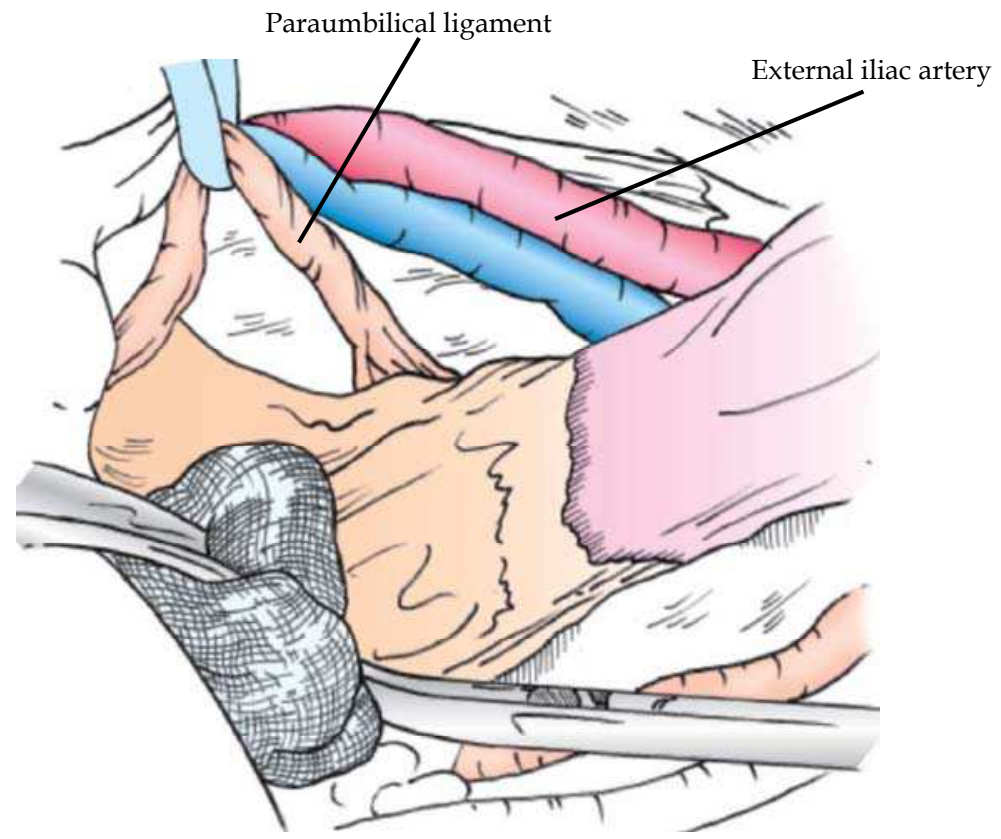

Fig. 10. Detachment of the urinary bladder 
to the vicinity of the uterine artery when detaching the bladder, it will facilitate the subsequent procedure involving the anterior layer of the vesicouterine ligament. Bleeding occurring during detachment of the bladder disturbs localization of the vesicouterine pouch, often resulting in injury to the muscular layer of the uterine cervix or the muscular layer of the bladder. Detachment of the uterus from the bladder is extremely important and requires good manipulation of the non-resistive part with gauze, a side plate, and Cooper scissors. The injured muscular layer of the bladder, if any, should be sutured with Surgisorb 3.0.

\subsubsection{Clamping, cutting, and ligation of the uterine artery}

When adipose tissue in the periphery of the paraumbilical ligament is removed, the vesical artery, and then the uterine artery, become visible. The uterine artery bifurcates into the ascending and descending branches after going beyond the ureter and entering the uterus. To preserve the feeding vessels distributed over the ureter, the main trunk of the uterine artery before the bifurcation beyond the ureter should be cut (Fig. 11).

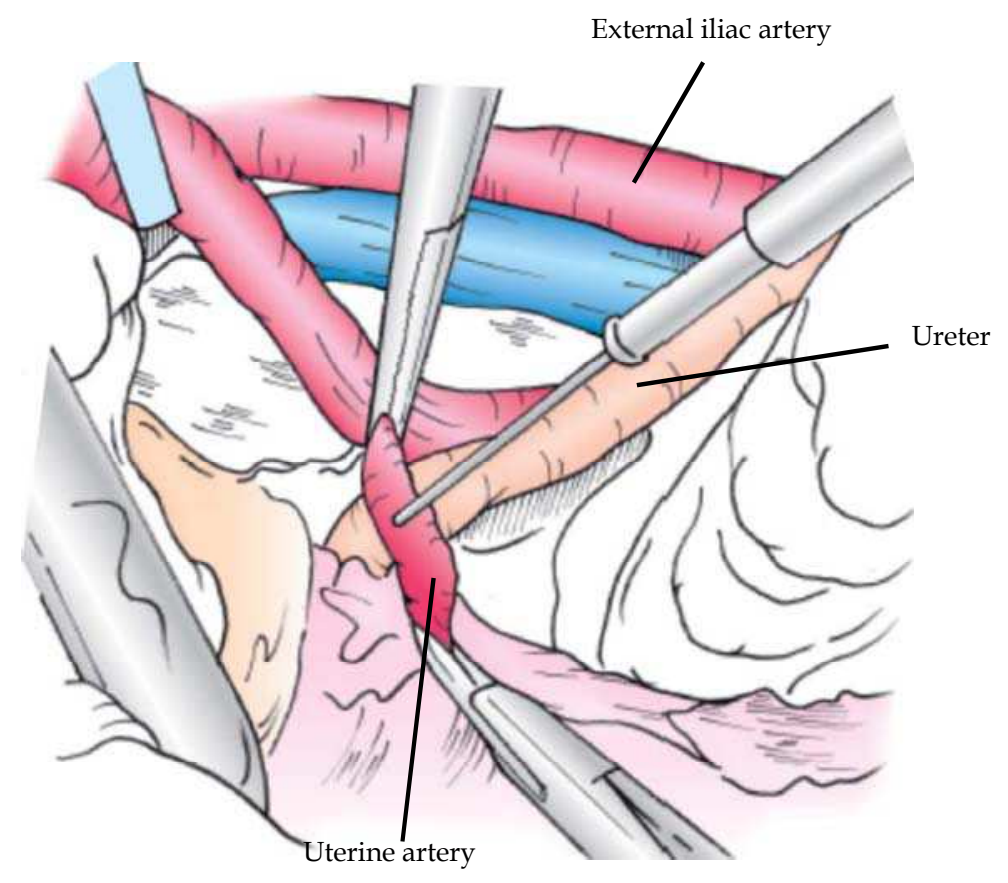

Fig. 11. Clamping, cutting, and ligation of the uterine artery

\subsubsection{Dissection of the anterior layer of the vesicouterine ligament}

While pulling the ureter with a ureteral retractor, the orifice portion of the ureter is displaced laterally with Cooper scissors. Then, a ureteral tunnel is formed by further lateral manipulation of Cooper scissors 2-3 times in a direction parallel to the course of the ureter while clamping the anterior layer of the vesicouterine ligament with forceps (Fig. 12). 


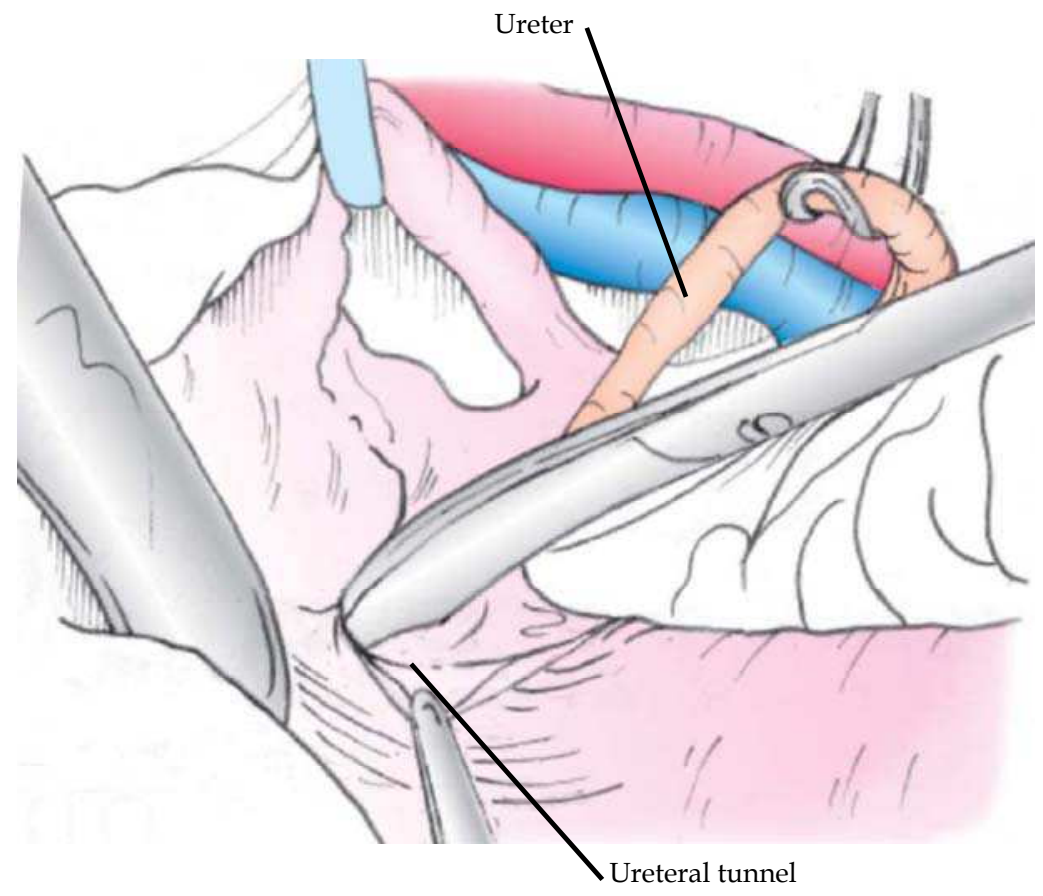

Fig. 12. Formation of a ureter tunnel.

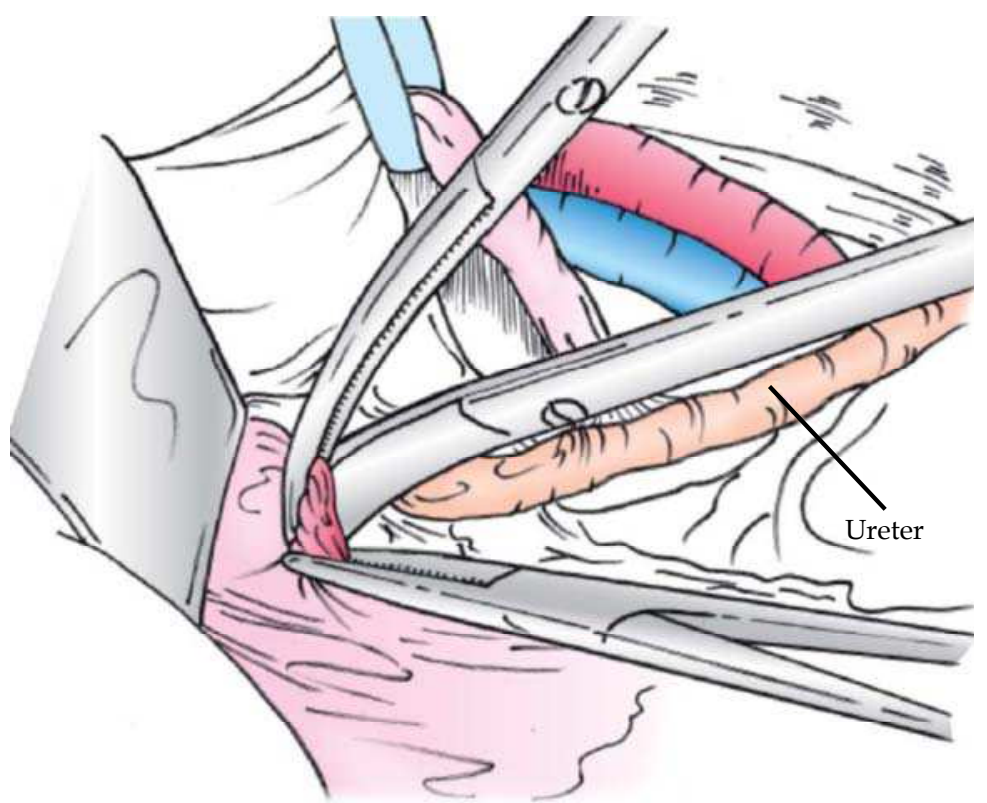

Fig. 13. Clamping, cutting and ligation of the anterior layer of the vesicouterine ligament. 
Formation of a ureteral tunnel is smooth if the ureter is displaced laterally in parallel to the course of the ureter with Cooper scissors, making use of the small space made by elevation of the anterior layer of the vesicouterine ligament with tweezers. Then, while holding the laterally displaced ureter in the ureteral tunnel with Cooper scissors, the anterior layer of the vesicouterine ligament is clamped with Kelly forceps necessarily set perpendicular to the uterine axis (Fig. 13). Then, the anterior layer of the vesicouterine ligament on the uterine side is clamped with a short and straight Kocher forceps. At this time, it is necessary to exercise caution against clamping of the ureter. Then, the anterior layer of the vesicouterine ligament on the uterine side is clamped with a short and Kocher forceps and cut with an electric knife. A single procedure is usually adequate, but two divided procedures of cutting the anterior layer may be employed if the dissection of the anterior layer is difficult.

\subsubsection{Detachment of the ureter in the posterior layer of the vesicouterine ligament}

Although the ureter running in the posterior layer of the bladder is exposed, the lower portion of the ureter is adherent to the posterior layer of the vesicouterine ligament with sparse connective tissue. Therefore, the ureter in the posterior layer can be easily isolated from the uterine side if the ureter is displaced laterally with Cooper scissors from above with rolled gauze set in place (Fig. 14). No processing of the posterior layer of the vesicouterine ligament is necessary.

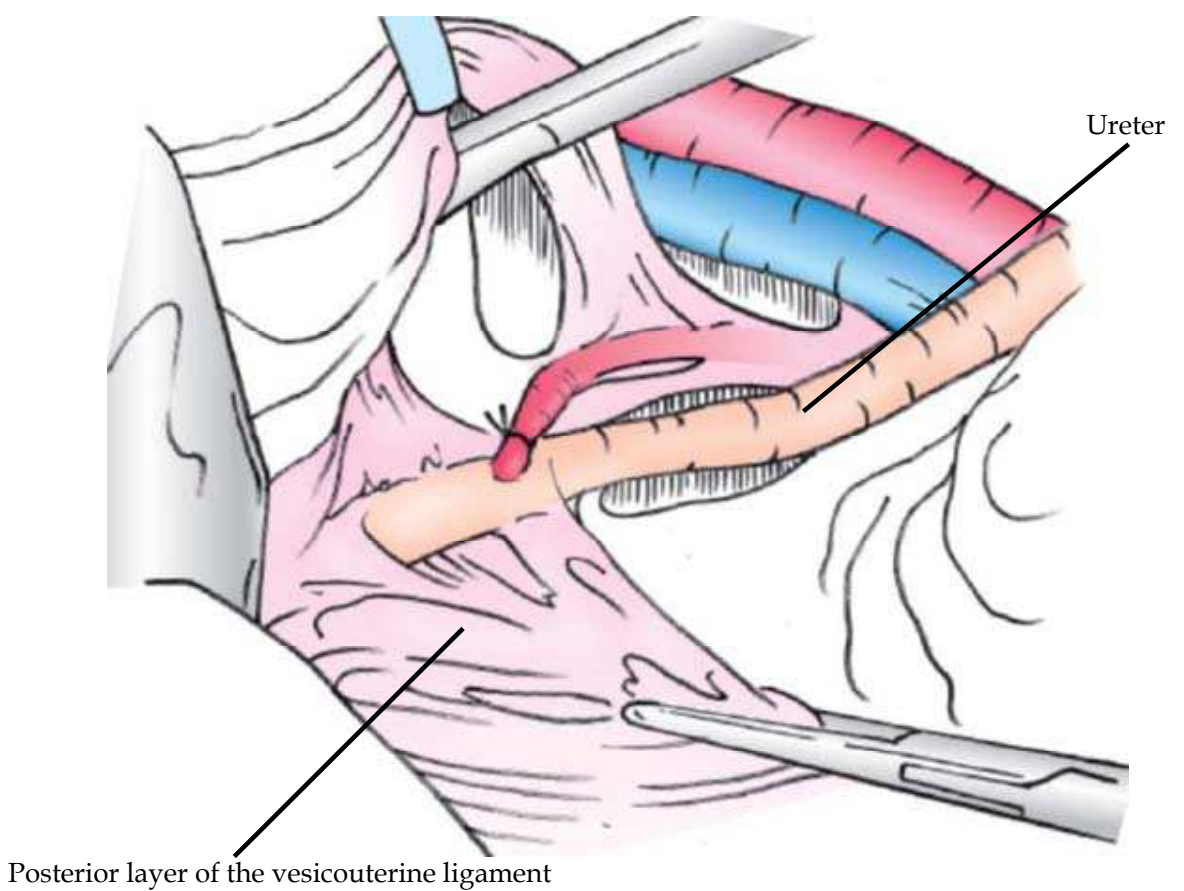

Fig. 14. Detachment of the ureter in the posterior layer of the vesicouterine ligament. 


\subsubsection{En bloc clamping, cutting and ligation of the posterior layer of the vesicouterine ligament, cardinal ligament and deep layer of the sacrouterine ligament}

When the bladder is displaced downward with a bladder retractor, and the uterus is adequately pulled upward, the ureter can be confirmed in a far lower location. After the positional relation among the muscular layer of the uterine cervix, bladder, vaginal wall and the course of the ureter is confirmed by palpation, the posterior layer of the vesicouterine ligament, the cardinal ligament and the deep layer of the sacrouterine ligament are clamped en bloc with the versatile forceps, and a short and straight Kocher forceps is also used on the uterine side to prevent backflow of blood (Fig. 15). To prevent bleeding, the tissues are cut with an electric knife at a site slightly beyond the portion clamped with the versatile forceps to allow a safe margin, and sutured with a needle and \#1 silk thread. At this time, the clamping area should be palpated before clamping to ensure that the muscular layer of the uterine cervix is not held with the versatile forceps. Because the ureter is detached sufficiently from the uterine side, a single clamping procedure is adequate to avoid injury to the ureter.

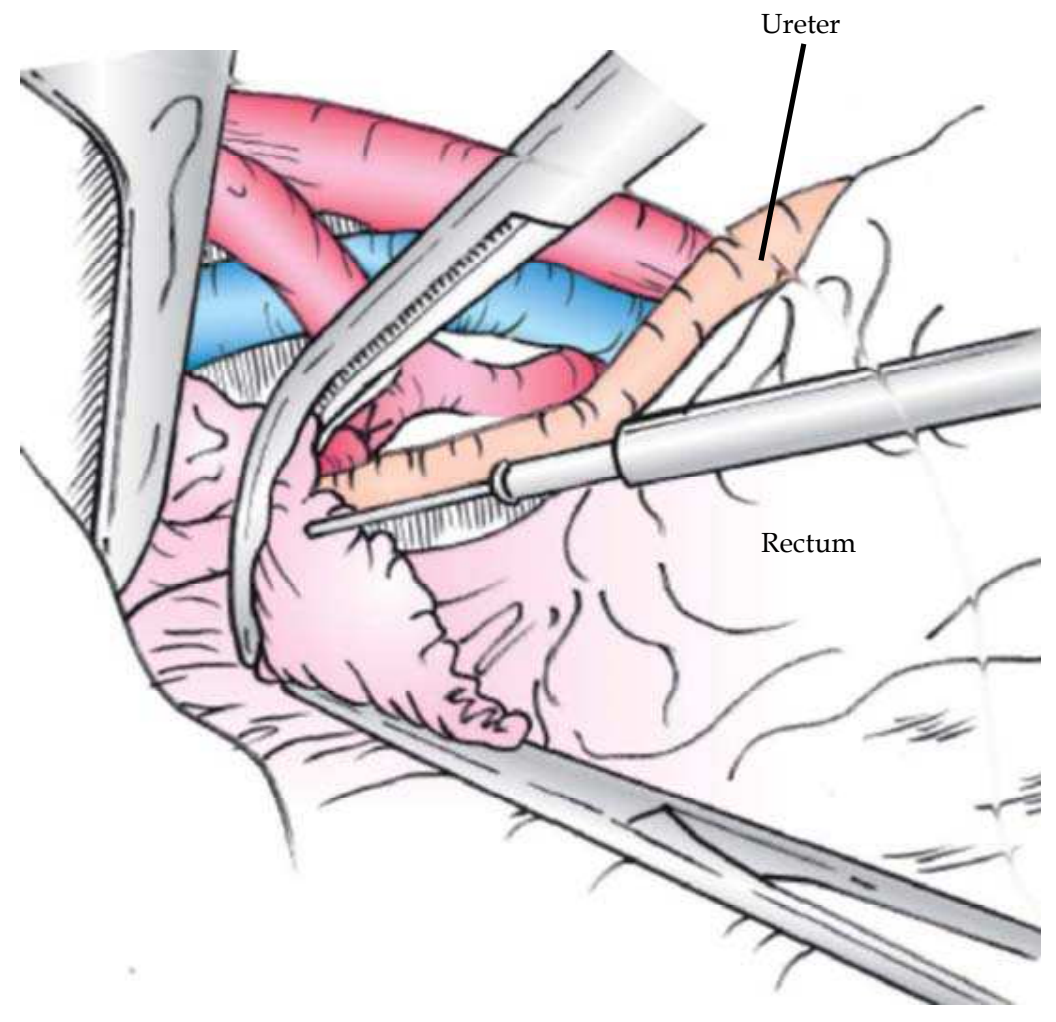

Fig. 15. En bloc clamping, cutting and ligation of the posterior layer of the vesicouterine ligament, cardinal ligament and deep layer of the sacrouterine ligament. 


\subsubsection{Clamping, cutting, and ligation of the paravaginal connective tissue}

Then, the remaining paravaginal connective tissue is clamped with the versatile forceps and cut, while setting the short and straight Kocher forceps on the uterine side. By this procedure, the vaginal canal is completely isolated to allow sufficient resection of the vaginal wall.

\subsubsection{Cutting of the vaginal canal and removal of the uterus}

After clamping the bilateral cut ends of the vaginal wall with the curved Kocher forceps to avoid slippage, a towel gauze is placed in the Douglas' pouch, and the bladder is displaced downward sufficiently with a bladder retractor. The uterus is then elevated, and the cutting site is confirmed by palpation to avoid cutting into the uterine cervix, and the vaginal wall is clamped with the large and curved Kocher forceps to prevent any escape of endometrial carcinoma tissue (Fig. 16). The vaginal wall in the vaginal convexity is cut with an electric knife, and the inside of the vagina is disinfected with an Isodine cotton swab. After gauze is inserted into the vagina to prevent leakage of intravaginal secretory fluid, the vaginal wall is clamped, held, and pulled with the long and straight Kocher forceps while incising the vaginal wall.

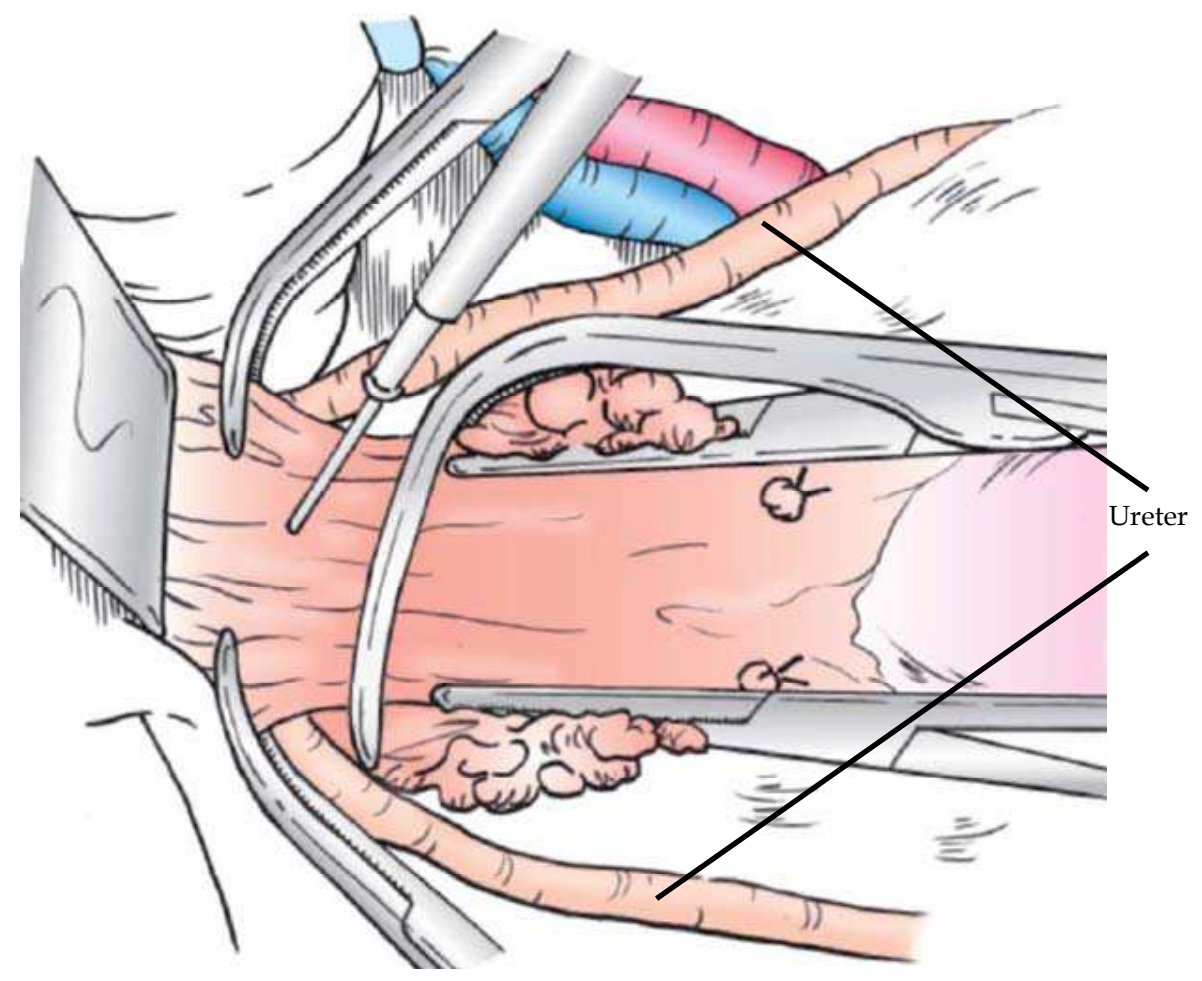

Fig. 16. Cutting of the vaginal canal and removal of the uterus. 


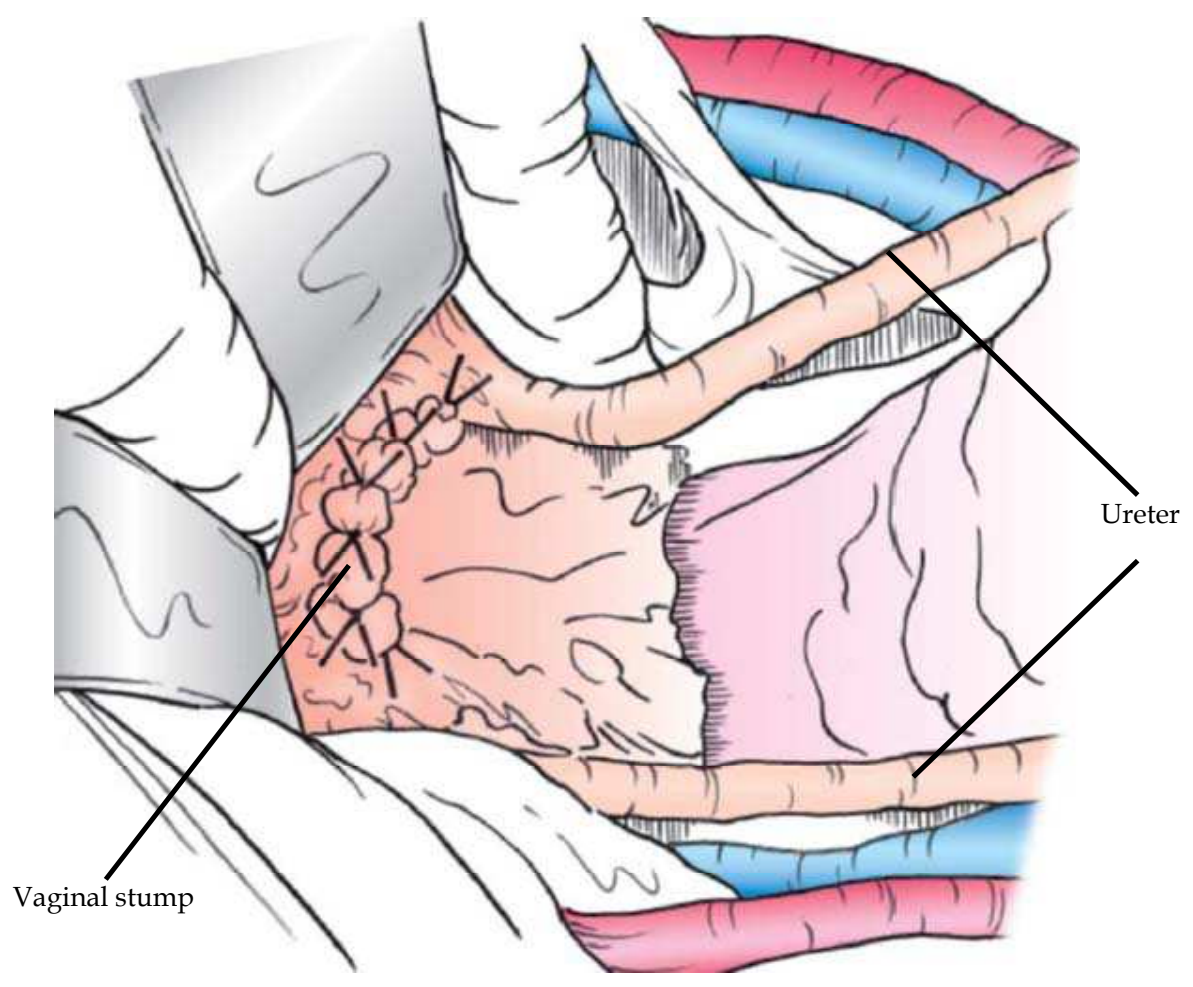

Fig. 17. Vaginal wall suture.

\subsubsection{Vaginal wall suture}

First, the bilateral vaginal wall cut ends are subjected to simple suture ligation with a blunt needle, 1-0 control release Vicryl, and cut, and the anterior and posterior portions of the vaginal wall are sutured by $Z$ suture and pulled (Fig. 17). Because bleeding is likely to occur from the bilateral cut ends of the vaginal wall, particular caution is required. Taking into consideration prevention of shortening of the vaginal canal and the patient's QOL, including the postoperative sex life in individual cases, the cut ends of the vaginal wall are opened and sutured in a continuous fashion to keep the length of the vagina. When lymphadenectomy is performed, the retroperitoneum should not be sutured to allow for smooth drainage of lymph, and a closed-end drain should be inserted through the abdominal wall and retained in the pelvic floor. 


\subsubsection{Confirmation of hemostasis and abdominal wall suture}

After arrest of bleeding from the posterior aspect of the detached bladder, the cut end suture site on the vaginal wall, en bloc cut ends of the vesicouterine ligament, cardinal ligament, and deep layer of the sacrouterine ligament, the cut end of the sacrouterine ligament, inside the pelvic cavity, and the cut sites is confirmed, double ligation of the bilateral infundibulopelvic ligament and round ligament of the uterus is performed. In cases of bleeding from the connective tissue, $\mathrm{Z}$ sutures with 3-0 silk thread are employed, and the simple suture thread of the vaginal cut ends is cut off. The pelvic peritoneum is opened, and Seprafilm ${ }^{\circledR}$ is attached to the bilateral retroperitoneal spaces for preventing adhesion. After putting the rectum back in its original position, the laparotomy incision is closed. The gauze count, including towel gauze, is confirmed by the doctors and nurses before closure of the incision. For closure of the laparotomy incision, the peritoneum is sutured continuously with a 2-0 Surgisorb cutting needle, and the cut ends of the fascia are sutured at 2 or 3 sites by a simple ligation suture with \#1 Surgilon. The fascia should be sutured continuously with a \#1 Polysorb blunt needle, while simple ligation is carried out at 2 or 3 sites to prevent abdominal wall hernia. The skin is closed by simple ligation suture by the mattress suture technique with 1-0 nylon thread, with the skin edges between the ligations are approximated with a Steri-strip. Finally, the vaginal wall is retracted with a vaginal speculum to remove gauze, and absence of bleeding from the vaginal wall cut ends is confirmed. The operation is ended after the absence of any remaining gauze is confirmed on a plain radiograph taken just before the end of the surgical procedure.

\section{Materials and methods}

Between December 1987 and December 2002 we performed modified radical type II hysterectomy with bilateral salpingo-oophorectomy including systematic pelvic and paraaortic lymphadenectomy, and peritoneal cytology in 284 endometrial carcinoma patients according to the classification of the International Federation of Gynecology and Obstetrics (stage IA, n=66; stage IB, $n=96$; stage IC, $n=33$; stage IIA, $n=5$; stage IIB, $n=20$; stage IIIA, $\mathrm{n}=28$; stage IIIC, $\mathrm{n}=28$; and stage IV, $\mathrm{n}=8$, Announcements FIGO stage, 1989) who gave informed consents at our institute. Systematic pelvic and para-aortic lymphadenectomy included complete dissection of lymph nodes from the femoral ring caudally to the lower margin of the renal vessels. No patients had intraabdominal residual lesions after surgery. Other inclusion criteria were as follows: Eastern Cooperative Oncology Group (ECOG) performance status of 0 to 2, age 75 years or younger, normal bone marrow, renal, liver, and cardiac function. The mean age (range) was 56 (26-75) years, with the median follow-up being 70 (24-189) months, and there were 88 and 196 pre- and postmenopausal patients, respectively. Histological examination showed 257 endometrioid adenocarcinomas (grade 1, $\mathrm{n}=129$; grade 2, $\mathrm{n}=101$, grade $3, \mathrm{n}=27$ ), 16 adenosqamous carcinomas, 8 serous adenocarcinomas and 3 clear cell adenocarcinomas. The patients with a tumor confined to the uterus (stage IC and II) for intermediate group were treated by 3 courses of CEP (cyclophosphamide $750 \mathrm{mg} / \mathrm{m}^{2}$, epirubicin $50 \mathrm{mg} / \mathrm{m}^{2}$, and cisplatin $75 \mathrm{mg} / \mathrm{m}^{2}$ ) regimen $3-4$ weeks apart and patients with intrapelvic lesions involving adnexa and/or pelvic lymph node (PLN) for high risk group were treated by 5 courses. In addition, 10 courses were given in the patients with extrapelvic lesions involving para-aortic lymph node (PAN) for 
high risk group. We have no indication for adjuvant radiotherapy in patients with endometrial carcinoma in this protocol. These anti-cancer agents were administered intravenously on Day 1. Univariate analysis for recurrence risk factors was performed by the $\mathrm{X}^{2}$ test. Recurrence risk factors for endometrial carcinoma ware analyzed for multivariate logistic regression using StatView (SAS Institute Inc.) Version 5.0. P-values of less than 0.05 were considered to be statistically significant.

\section{Results and discussion}

The overall incidence of retroperitoneal lymph node (RLN) metastasis assessed by systematic pelvic and para-aortic lymphadenectomy was $12.0 \%(34 / 284)$ in Stage I-IV endometrial carcinoma, and incidences of PLN and PAN metastasis were 9.2\% (26/284) and $7.4 \%(21 / 284)$, respectively. However, PAN metastasis rate is $50 \%(13 / 26)$ in patients with PLN metastasis. Thirteen patients $(4.6 \%)$ had lymph node metastasis in the pelvic region alone, $8(2.8 \%)$ in the para-aortic region alone and $13(4.6 \%)$ in both regions. This low incidence of isolated PAN metastasis was possibly considered due to many early stage I diseases, and para-aortic lymphadenectomy should be performed in high risk patients with intrapelvic extention, for example, PLN metastasis or ovarian metastasis. We are now performing systematic pelvic lymphadenectomy and resect in clinically suspicious PAN metastasis for high risk patients. The incidence of RLN metastasis by histological examination was $6.2 \%(8 / 129)$ in grade $1,12.0 \%(12 / 101)$ in grade $2,18.5 \%(5 / 27)$ in grade 3 , $25.0 \%(4 / 16)$ in adenosquamous carcinoma, $50.0 \%(4 / 8)$ in serous adenocarcinoma and $33.3 \%(1 / 3)$ in clear cell adenocarcinoma. The incidence of lymph node metastasis was lower in endometrioid adenocarcinoma grade 1 than in endometrioid adenocarcinoma grade 2/3, and there was also a high incidence of lymph node metastasis in the histological subtypes, so-called adenosquamous carcinoma, serous carcinoma, and clear cell adenocarcinoma. The average number (range) of dissected nodes was 24.7 (10-62) for PLN and 10.1 (5-47) for PAN. We had more of operating time as well as blood loss for this operation, but this is clinically within permissible limits because there was no need to give a blood transfusion to the patients . Operating time and blood loss (mean average \pm standard deviation: SD), in modified radical hysterectomy including systematic pelvic and para-aortic lymphadenectomy, were $222 \pm 61.6 \mathrm{~min}$ and $545 \pm 301 \mathrm{ml}$, respectively. There was no severe lymphedema associated with systematic lymphadenectomy.

One of the main postoperative adjuvant treatments is radiation and vaginal brachytherapy, however, there are few papers dealing with long-term survival in patients with systematic retroperitoneal lymphadenectomy followed by postoperative adjuvant chemotherapy. PAC (cisplatin, doxorubicin and cyclophosphamide) is one of the most commonly used regimens in endometrial carcinoma, and the less cardiotoxic analog epirubicin seemed to have the same activity as doxorubicin. Gadducci et al., (1999) described that the combination of cisplatin, epirubicin, and cyclophosphamide had good activity in advanced or recurrent endometrial carcinoma. Therefore, we examined recurrence risk factors over the past 10 years in patients with surgically staged endometrial carcinoma followed by postoperative CEP regimen. Postoperative adjuvant CEP chemotherapy was performed in 58 patients with a tumor confined to the uterus (Stages IC and II) in 3 courses 3-4 weeks apart and in 43 patients with extrauterine lesions involving adnexa and/or PLN in 5 courses, and also in 21 
patients with PAN metastasis in 10 courses. No patient required major modification of the treatment modality of the postoperative adjuvant chemotherapy because of acute myelosuppression or gastrointestinal disorder (Hiura et al., 2010). Gynecologic Oncology Group (GOG) randomized trial (Randall et al., 2006) showed that AP (doxorubicin and cisplatin) chemotherapy significantly improved progression-free and overall survival compared with WAI (whole-abdominal irradiation) in patients with Stages III or IV endometrial carcinoma with a maximum of $2 \mathrm{~cm}$ of postoperative residual disease. Paclitaxel (Lissoni et al., 1996) is active in patients with endometrial cancer pretreated with PAC, and also carboplatin (Burke et al., 1993) has definite activity in endometrial carcinoma and offers a well-tolerated palliative therapeutic alternative. Phase III randomized study of doxorubicin, cisplatin, paclitaxel, and filgrastin (G-CSF) versus carboplatin and paclitaxel in patients with stage III or IV or recurrent endometrial cancer (GOG 209) is now analyzing (National Cancer Institute, 2011).

Recurrence was detected in $20(7.0 \%)$ cases (vaginal stump: 2, pelvic cavity: 2, external lymph node: 1, pelvic cavity + vaginal stump: 1, lung: 7, pleura: 1, PAN: 2, liver + abdominal cavity: 1 , bone: 1 , ascites: 1 , pelvic cavity + abdominal cavity: 1 ) with a median

\begin{tabular}{clll}
\hline Stage & Local & Distant & Local + Distant \\
\hline & & Lung (2) & \\
I/II & Vaginal stump (1) & Pleura(1) & \\
$(5)$ & & PAN (1) & \\
& & Lung (5) & Pelvic cavity + Abdominal \\
& Vaginal stump (1) & Liver + Abdominal & cavity (1) \\
III/ IV & Pelvic cavity (2) & cavity (1) & \\
$(15)$ & Pelvic cavity + Vaginal & PAN (1) & \\
& stump(1) & Bone (1) & Ascites (1) \\
& &
\end{tabular}

Recurrence rate :7\%(20/284), PAN:Para-aortic lymph node, LN: Lymph node

Table 1. Sites of recurrence in endometrial carcinoma

\begin{tabular}{cc}
\hline & CR; \\
& Lung (1), PAN (1), Vaginal stump (1) \\
Pelvic cavity + Vaginal stump (1) & PR: Pelvic cavity (2), External LN (1), PAN(1), \\
& Liver + Abdominal (1) \\
SD: Lung (1), Pleura (1), Pelvic cavity + Abdominal cavity (1) & Vaginal stump (1) \\
SD (4) + PD (2) & PD: Lung (2) \\
\hline
\end{tabular}

Response rate: 9/15 (60\%), PAN: Para-aortic lymph node, LN: Lymph node,

CR: Complete response, PR: Partial response, SD: Stable disease, PD: Progressive disease

Table 2. Response rate in recurrent endometrial carcinoma 
disease-free interval from initial surgery of 689 days. One vaginal stump recurrence $(0.5 \%)$ in stage I/II and six cases of locoregional recurrence (vaginal stump: 1, pelvic cavity: 2, external lymph node: 1 , pelvic cavity + vaginal stump: 1 , pelvic cavity + abdominal cavity: 1 , $9.4 \%$ ) in stage III/IV were recognized (Table. 1). There were four cases of distant recurrence $(1.8 \%)$ in stage I/ II. The incidence of local recurrence in stage I/II was extremely lower more than expected. The response rate to chemotherapy or radiotherapy for recurrent diseases was 60.0 (9/15) \% (Table. 2). Six cases of locoregional recurrence and nine cases of distant recurrence were treated by radiotherapy and chemotherapy, respectively. Disease control rate (complete response: $\mathrm{CR} /$ particular response: $\mathrm{PR} /$ stable disease: $\mathrm{SD}$ ) showed $86.7 \%(13 / 15)$. The response rate to chemotherapy or radiotherapy for recurrence disease was comparatively good. The incidence of recurrence by histological examination was $2.3 \%$ $(3 / 129)$ in grade I, $8.9 \%(9 / 101)$ in grade II, $7.4 \%(2 / 27)$ in grade III, $12.5 \%(2 / 16)$ in adenosqamous carcinoma, $37.5 \%(3 / 8)$ in serous adenocarcinoma and $33.3 \%(1 / 3)$ in clear cell adenocarcinoma (Table 3). The incidence of recurrence was more lower in endometrioid adenocarcinoma grade I than in endometrioid grade 2/3, and there was a high incidence of recurrence in the histological subtypes, adenosquamous carcinoma, serous adenocarcinoma, and clear cell adenocarcinoma. Recurrence risk factors by univariate analysis were menopause $(p=0.0099)$, histology $(p=0.005)$, FIGO stage $(p<0.0001)$, myometrial invasion $(\mathrm{p}<0.0001)$, adnexal metastasis $(\mathrm{p}=0.0009)$, lymphvascular space invasion $(\mathrm{p}<0.0006)$, tumor diameter $(p=0.0076)$, peritoneal cytology $(p=0.039)$, and RLN metastasis $(p=0.0009)$ (Table 4$)$. Cervical involvement $(p=0.3092)$ was not recognized as a recurrence risk factor. A multivariate analysis showed that menopause $(p=0.029)$ and FIGO stage $(p=0.0369)$ were the most significant predictors of recurrence (Table 5). The careful follow-up is always required in endometrial carcinoma with the independent risk factors including menopause and FIGO stage III/IV.

\begin{tabular}{lcc}
\hline \multicolumn{1}{c}{ Histological type } & No. of Patients & Incidence \\
\hline Endometrial adenocarcinomas & 129 & 3 \\
Grade I & 101 & $(2.3)$ \\
Grade II & & 9 \\
& 27 & $(8.9)$ \\
Grade III & & 2 \\
& 16 & $(7.4)$ \\
Adenosquamous carcinomas & & 2 \\
& 8 & $(12.5)$ \\
Serous adenocarcinoma & 3 & 3 \\
Clean cell adenocarcinoma & & $(37.5)$ \\
\end{tabular}

Table 3. Incidences of recurrence by histological examination 


\begin{tabular}{|c|c|c|c|}
\hline & $\begin{array}{l}\text { No. of Patients } \\
\text { Total }(\mathrm{N})\end{array}$ & Recurrence (\%) & p-value \\
\hline \multicolumn{4}{|l|}{ Menopause } \\
\hline Premenopause & 87 & $1(1.5)$ & 0.0099 \\
\hline Postmenopause & 197 & $19(9.6)$ & \\
\hline \multicolumn{4}{|l|}{ Histology } \\
\hline G1 & 129 & $3(2.3)$ & 0.005 \\
\hline G2, 3 and others & 155 & $17(10.9)$ & \\
\hline \multicolumn{4}{|c|}{ Cervical involvement } \\
\hline Negative & 247 & $16(6.5)$ & 0.3092 \\
\hline Positive & 37 & $4(10.8)$ & \\
\hline \multicolumn{4}{|l|}{ FIGO stage } \\
\hline I / II & 220 & $5(2.3)$ & $<0.0001$ \\
\hline III/ IV & 64 & $15(23.4)$ & \\
\hline \multicolumn{4}{|c|}{ Myometrial invation } \\
\hline$\leqq 1 / 2$ & 201 & $5(2.5)$ & $<0.0001$ \\
\hline$>1 / 2$ & 83 & $15(18.1)$ & \\
\hline \multicolumn{4}{|c|}{ Adenexal metastasis } \\
\hline Negative & 265 & $14(5.3)$ & 0.0009 \\
\hline Positive & 19 & $6(31.6)$ & \\
\hline \multicolumn{4}{|c|}{ Lymph vascular space invasion } \\
\hline Negative & 166 & $4(2.4)$ & $<0.0006$ \\
\hline Positive & 118 & $16(13.6)$ & \\
\hline \multicolumn{4}{|l|}{ Tumor diameter } \\
\hline$\leqq 4 \mathrm{~cm}$ & 171 & $6(3.5)$ & 0.0076 \\
\hline$>4 \mathrm{~cm}$ & 113 & $14(12.4)$ & \\
\hline \multicolumn{4}{|l|}{ Peritoneal cytology } \\
\hline Negative & 251 & $13(5.2)$ & 0.039 \\
\hline Positive & 33 & $7(21.2)$ & \\
\hline \multicolumn{4}{|l|}{ RLN metastasis } \\
\hline Negative & 258 & $14(5.4)$ & 0.0009 \\
\hline Positive & 26 & $6(31.6)$ & \\
\hline
\end{tabular}

RLN: Retroperitoneal lymph node

Table 4 . Recurrence risk factors by univariate analysis in endometrial carcinoma 


\begin{tabular}{lccc}
\hline & p-value & Odd ratio & $95 \%$ CI \\
\hline $\begin{array}{l}\text { Menopause } \\
\quad \text { Premenopause/ Postmenopause }\end{array}$ & 0.029 & 9.553 & $1.295-72.449$ \\
$\begin{array}{l}\text { Histology } \\
\quad \text { G1/G2, 3 and others }\end{array}$ & 0.0663 & 3.253 & $0.923-11.460$ \\
$\begin{array}{l}\text { FIGO stage } \\
\quad \text { I, II/III, IV }\end{array}$ & 0.0369 & 4.017 & $1.088-14.830$ \\
$\begin{array}{l}\text { Myometrial invasion } \\
\quad 1 / 2 />1 / 2\end{array}$ & 0.2452 & 1.945 & $0.633-5.974$ \\
$\begin{array}{l}\text { Lymph vascular space invasion } \\
\quad \text { Negative / Positive }\end{array}$ & 0.2128 & 2.079 & $0.657-6.576$ \\
$\begin{array}{l}\text { Tumor diameter } \\
\quad \text { 4cm / > 4cm }\end{array}$ & 0.1629 & 2.025 & $0.751-5.458$ \\
$\begin{array}{l}\text { RLN metastasis } \\
\text { Negative / Positive }\end{array}$ & 0.1773 & 2.245 & $0.671-7.265$ \\
\hline
\end{tabular}

CI: Confidence interval, RLN: Retroperitoneal lymph node.

Table 5. Recurrence risk factors by multivariate analysis in endometrial carcinoma

\section{Conclusion}

In modified radical hysterectomy, the uterus should be extirpated with the cardinal ligament allowing an extra $1.5-2.0 \mathrm{~cm}$ margin of the vaginal wall. The key point of the technique is formation of a ureteral tunnel during the dissection of the anterior layer of the vesicouterine ligament, while lightly pulling the ureter with tweezers, Cooper scissors and ureteral retractors. Modified radical hysterectomy has a broad range of applications, being positioned in between total hysterectomy and radical hysterectomy. This surgical technique has the advantage that postoperative urinary disturbances and other complications are minimized if the surgical candidates are selected appropriately. This surgical procedure could contribute to reduce locoregional recurrence, especially vaginal stump in stage I/II. It is suggested that management of endometrial carcinoma with risk factors by appropriate surgery and adjuvant chemotherapy is very important for preventing both locoregional and distant recurrence. Thus, further application of this technique is expected.

\section{Acknowledgments}

This study was supported by a grant from the Japanese Organization of the Ministry of Health, Labor and Welfare (2010).

\section{References}

Announcements FIGO stages - 1988 revision (1989). Gynecol Oncol. Vol.35, Issue 1, (Oct 1989), pp125-127. 
Bidus, MA. \& Elkas JC. (2007). Cervical and Vaginal Cancer, In: Berek \& Novak's Gynecology, I.S. Berek (Ed), pp.1403-1456, ISBN: 978-0781768054, Philadelphia, USA

Blake, P. Swart, AM. \& Orton, J. et al. (2008). Adjuvant external beam radiotherapy in the treatment of endometrial cancer (MRC ASTEC and NCIC CTG EN.5 randomised trials): pooled trial results, systematic review, and meta-analysis. Lancet, pp. 137-46, ISSN 0140-6736

Burke, TW. Munkarah, A \& Kavanagh, JJ. et al. (1993). Treatment of advanced or recurrent endometrial carcinoma with single-agent carboplatin. Gynecol Oncol. Vol. 51, (Dec 1993), pp.397-400, ISSN: 0090-8258

Creutzberg, CL.; van Putten, WL. \& Koper, PC. et al. (2000). Surgery and Postoperative Radiotherapy Versus Surgery Alone for Patients with Stage-1 Endometrial Carcinoma: Multicentre Randomised Trial. Lancet, Vol. 355, (April 2000), pp.14041411, ISSN 2078- 6891

Gadducci, A.; Romanini, A. \& Cosio, S. et al. (1999). Combination of cisplatin, epirubicin, and cyclophosphamide (PEC regimen) in advanced or recurrent endometrial cancer: a retrospective clinical study. Anticancer Res, Vol. 19, (May 1999), pp.22532256, ISSN:0250-7005

Hiura, M.; Nogawa, T. \& Matsumoto T. et al. (2010).Long-term Survival in Patients with Para-aortic Lymph Node Metastasis with Systematic Retroperitoneal Lymphadenectomy Followed by Adjuvant Chemotherapy in Endometrial Carcinoma. International Journal of Gynecological Cancer, Vol. 20, Issue 6, (August 2010), pp. 1000-1005, ISSN 1048-891X

Hiura, M.; Nogawa, T. (2011). Modified Radical Hystractomy in Endometrial carcinoma, In: Obstetric and Gynecologic Surgery NOW No.6. I. Konishi (Ed), 34-45, ISBN:978-4-75831205-9 C3347, Tokyo, Japan.

Jones III, HW (2008). Cervical Cancer Precursor and Their Management, In: Te Linde's Operative Gynecology, I.S. J.A. Rock \& H.W. Jones III(Eds), 1208-1290, ISBN: 9780781772341, Philadelphia, USA

Keys, HM.; Roberts, JA.\& Brunetto VL. et al. (2004).A phase III trial of surgery with or without adjunctive external pelvic radiation therapy in intermediate risk endometrial adenocarcinoma: a Gynecologic Oncology Group study. Gynecologic Oncology, Vol. 92, Issue 3, (March 2004), pp.744-751, ISSN 0090-8258

Lissoni, A, Zaneta, G \& Losa, G. et al. (1996). Phase II study of paclitaxel as salvage treatment in advanced endometrial cancer. Ann Oncol. Vol. 7, Issue 8, (Oct 1996), pp.861-863, ISSN: 0923-7534

National Cancer Institute. Phase III randomized study of doxorubicin, cisplatin, paclitaxel, and fligrastim (G-CSF) versus carboplatin snd paclitaxel in patients with stage III or VI or recurrent endometrial cancer.

http:/ / www.cancer.gov/clinicaltrials/search/view?cdrid=305940\&version=Healt hProfessional

National Comprehensive Cancer Network ${ }^{\circledR}$ (2011). NCCN Clinical Practice Guideline Oncology TM Uterine Neoplasms V.I. Available from http://www.nccn.org/professionals/physician_gls/f_guidelines.asp 
Nout, RA.; Smit, VT. \& Putter, H. et al. (2010). Vaginal Brachytherapy versus Pelvic External Beam Radiotherapy for Patients with Endometrial Cancer of High-intermediate Risk (PORTEC-2): An Open-label, Non-inferiority, Randomised Trial. Lancet, Vol.375, Issue 9717, (March 2010), pp. 816-823, ISSN 0140-6736

Randall, ME.; Filiaci, VL. \& Muss, H. et al. (2006). Randomized phase III trial of wholeabdominal irradiation versus doxorubicin and cisplatin chemotherapy in advanced endometrial carcinoma: a Gynecologic Oncology Group Study. J Clin Oncol, Vol. 24, (Jan 2006), pp.36-44, ISSN:0732-183X

Randall, ME.; Michael, H. Long III, H. \& Tedjanati, S. (2009). Uterine Cervix, In: Principles and Practice of Gynecologic Oncology, R.R.Barakat,, M. Markman \& M.E. Randall (Eds), 623-681, ISBN: 978-0781778459, Philadelphia, USA 


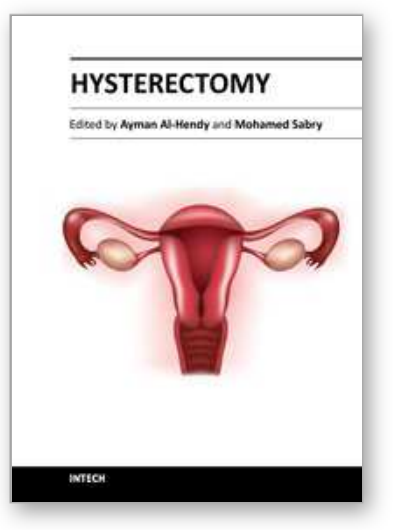

\author{
Hysterectomy \\ Edited by Dr. Ayman Al-Hendy
}

ISBN 978-953-51-0434-6

Hard cover, 426 pages

Publisher InTech

Published online 20, April, 2012

Published in print edition April, 2012

This book is intended for the general and family practitioners, as well as for gynecologists, specialists in gynecological surgery, general surgeons, urologists and all other surgical specialists that perform procedures in or around the female pelvis, in addition to intensives and all other specialities and health care professionals who care for women before, during or after hysterectomy. The aim of this book is to review the recent achievements of the research community regarding the field of gynecologic surgery and hysterectomy as well as highlight future directions and where this field is heading. While no single volume can adequately cover the diversity of issues and facets in relation to such a common and important procedure such as hysterectomy, this book will attempt to address the pivotal topics especially in regards to safety, risk management as well as pre- and post-operative care.

\title{
How to reference
}

In order to correctly reference this scholarly work, feel free to copy and paste the following:

Masamichi Hiura and Takayoshi Nogawa (2012). The Role of Modified Radical Hysterectomy in Endometrial Carcinoma, Hysterectomy, Dr. Ayman Al-Hendy (Ed.), ISBN: 978-953-51-0434-6, InTech, Available from: http://www.intechopen.com/books/hysterectomy/the-role-of-modified-radical-hysterectomy-in-endometrialcarcinoma

\section{INTECH}

open science | open minds

\author{
InTech Europe \\ University Campus STeP Ri \\ Slavka Krautzeka 83/A \\ 51000 Rijeka, Croatia \\ Phone: +385 (51) 770447 \\ Fax: +385 (51) 686166 \\ www.intechopen.com
}

\author{
InTech China \\ Unit 405, Office Block, Hotel Equatorial Shanghai \\ No.65, Yan An Road (West), Shanghai, 200040, China \\ 中国上海市延安西路65号上海国际贵都大饭店办公楼405单元 \\ Phone: +86-21-62489820 \\ Fax: +86-21-62489821
}


(C) 2012 The Author(s). Licensee IntechOpen. This is an open access article distributed under the terms of the Creative Commons Attribution 3.0 License, which permits unrestricted use, distribution, and reproduction in any medium, provided the original work is properly cited. 